\title{
Genetic Algorithm Applied to the Time-Series Landing Flight Path and Control Optimization of a Supersonic Transport
}

\author{
Masahiro Kanazaki \\ Tokyo Metropolitan University \\ 6-6, Asahigaoka, Hino \\ Tokyo, Japan 192-0065 \\ kana@tmu.ac.jp
}

\author{
Ryouta Saisyo \\ Tokyo Metropolitan University \\ 6-6, Asahigaoka, Hino \\ Tokyo, Japan 192-0065 \\ saisho-ryouta@ed.tmu.ac.jp
}

\begin{abstract}
A genetic algorithm (GA) which is a meta-heuristic approach was applied to optimize the landing flight path of a delta-winged supersonic transport (SST). However, at low speeds, particularly during take-off and landing, a complex flowfield surrounds the delta wing. This phenomenon requires time-series control optimization that yields an optimum control sequence by aerodynamic - flight dynamics with high-fidelity computational fluid dynamics to evaluate the flight path with the complex flowfield. To this end, we presented an efficient flight simulation based on Kriging-model-assisted aerodynamic estimation to carry out the global optimization via a GA. After establishing the efficient aerodynamics-flight dynamics optimization, we constructed the design of the flight and control sequence for the time-series optimization of an effective SST landing. Several solutions that provide an allowable SST landing performance, along with the knowledge on optimum flight and control sequence, are presented herein.
\end{abstract}

\section{CCS Concepts}

- Applied computing Aerospace

\section{Keywords}

Genetic Algorithm, Kriging Model, Aerodynamics - Flight Simulation, Supersonic Transport, Time-Series Optimization, Real-World Application

\section{INTRODUCTION}

A supersonic transport (SST) system, i.e., the Concorde, often has a main wing with a large sweepback angle, such as the delta wing, to reduce aerodynamic drag at a supersonic cruise. Although such a wing configuration may effectively decrease the aerodynamic drag at high speeds, it may prove to be a problem while gaining sufficient lift force at low speeds, particularly during take-off and landing, where an SST requires a large angle of attack, but not too large to create a "stall," or the instance of losing lift owing to vortex breakdown [Ericsson 1996]. Safety during take-off

Permission to make digital or hard copies of all or part of this work for personal or classroom use is granted without fee provided that copies are not made or distributed for profit or commercial advantage and that copies bear this notice and the full citation on the first page. To copy otherwise, or republish, to post on servers or to redistribute to lists, requires prior specific permission and/or a fee.

ISMSI 2019, March 23-24, 2019, Male, Maldives

2019 Association for Computing Machinery

ACM ISBN 978-1-4503-7211-4/19/03 ..\$15.00

DOI: https://doi.org/10.1145/3325773.3325789 and landing is one of the serious factors considered in the manufacture of a civil aircraft, and is strongly related to its aerodynamic characteristics at a low-speed flight. Several studies on flowfields at low speeds around an SST [Ericsson 1996, Liu et al. 2018, Srigrarom and Lewpiriyawong 2007] have been performed; nevertheless, only a few have dealt with optimization of the flight and control sequence due to expensive aerodynamic evaluation cost for such complex flows.

In principle, the altitude, air speed, attitude, and atmospheric pressure should be time-serially varied with the aircraft flight. Moreover, evaluation of the flight path requires a combination of flight dynamics and aerodynamic tests. Additionally, flow phenomena around the delta wing at lowspeed, including the vortex and the separation, are characteristically complex, which necessitates a high-fidelity flow simulation that could be too costly even with a supercomputer. On this regard, the efficient aerodynamics - flight dynamics with aerodynamic force estimation [Kanazaki and Othmam 2016] from a given database, may be a promising alternative. For this approach, we introduced Kriging model of aerodynamic estimation during flight calculations. To validate its efficiency and accuracy, we conducted a low-fidelity flow flight simulation for a typical civil aircraft geometry. Both the numerical and simulated results agreed with each other.

We employ this approach to obtain knowledge on the efficient landing flight path and time-series control sequence of an SST. Initially, we determine the aerodynamic coefficients using a high-fidelity Reynolds-averaged Navier-Stokes (RANS) flow simulation at different aircraft attitudes and air speeds to develop a database of aerodynamic forces. Next, we estimate these forces through the application of the equations of motion (EoM) using the Kriging method. Only the lateral motion for the EoM, at 3 degrees-of-freedom (DoF), is considered. Results of the flight calculation reveal that the input of the steering angle is optimized by the genetic algorithm. Based on the above procedure, the optimization technique can search an optimal flight route for a supersonic aircraft based on a detailed and high-fidelity aerodynamic evaluation of the steering angle. To define the control procedure, many time-series parameter for control surfaces should be considered. Thus, we applied a genetic algorithm (GA) which is a meta-heuristic search, because it can help to discover the global knowledge unlike by the gradient based optimization. 


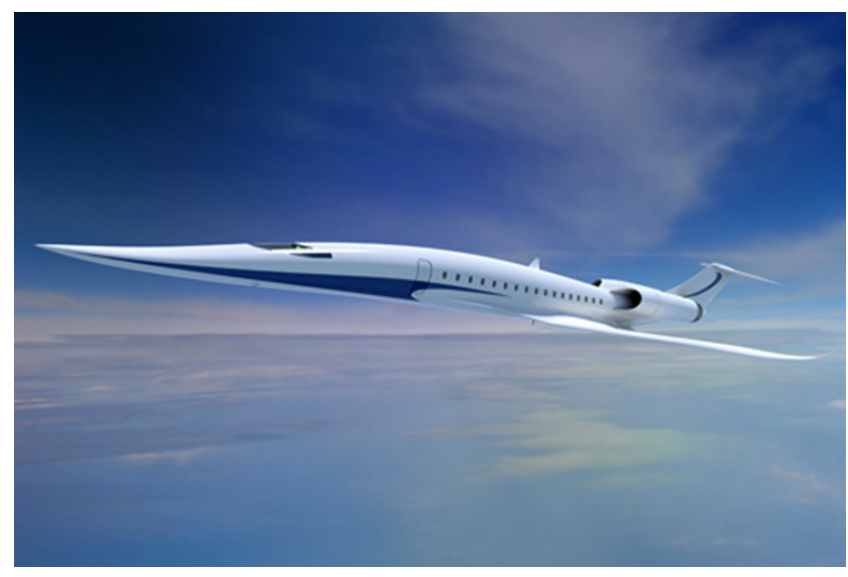

Figure 1: Illustration of SST concept, supersonic business jet by JAXA.

\section{DESIGN PROBLEM STATEMENT}

\subsection{Aircraft Model}

In this study, we dealt with the SST model conceptually designed by JAXA, as shown in Fig. 1. We constructed a simplified model, as shown Fig. 2, and excluded the engines in the aerodynamics evaluation. Adjusting the horizontal tail wing of this SST changes its attitude angle, which in turn, varies the aerodynamic drag and lift.

\subsection{Formulation}

The aircraft flight path was optimized by the cost function $J$ [18] as follows:

$$
J=\phi\left[x\left(t_{\mathrm{t}}\right), \delta e\left(t_{\mathrm{t}}\right)\right]+\int_{t_{0}}^{t_{\mathrm{t}}} L[x(t), \delta e(t)] d t .
$$

where $t$ indicates the flight time and the subscript $\mathrm{t}$ represents the period at which aircraft flight terminates at an aircraft height $(z(t))$ of $0.0[\mathrm{~m}]\left(z\left(t_{r m t}\right)=0.0\right) ; x(t)$ and $\delta e(t)$ are the flight distance and the control surface (horizontal tail wing) angle at $t$, respectively; the first term $\phi\left[x\left(t_{\mathrm{f}}\right)\right]$ is a function denoting the path angle of at the flight termination, and $L[x(t), \delta e(t)]$ can be expressed as follows:

$$
\begin{array}{rc}
L[x(t), \delta e(t)] & (2) \\
= & 2.0\left[\left(z(t)-z(0) \cos \alpha_{0}\right)-\left(x(t)-x(0) \sin \alpha_{0}\right)\right]^{2} \\
+ & 0.04[\delta e(t)-\delta e(0)]^{2}
\end{array}
$$

If $J$ obtained by Eq. 1 was minimum, then the aircraft landing path should be the most efficient.

To satisfy the terminal condition for the landing, the aircraft's vertical speed $w(t)$ was presumed to be than $5.0[\mathrm{~m} / \mathrm{s}]$ as a constraint. Thus, we solved the design problem:

$$
\left\{\begin{array}{l}
\text { Minimize } \quad J \\
\text { Subject to } w\left(t_{\mathrm{t}}\right)<5.0
\end{array}\right.
$$

A time-series variation of $\delta e(t)$ from $t=0[s]$ to $t=t_{\mathrm{t}}[s]$, at $0.2[\mathrm{~s}]$-intervals, was considered as the design variable. Thus, the possible range of $\delta e(t)$ in 1.0 [s.] was 0.5 [ $^{\circ}$

\subsection{Aerodynamic Flight Simulation Conditions}

The horizontal tail wing acted as a control surface for the aircraft flight, while the time-series $\delta e(t)$ variation controlled the pitch angle $\theta(t)$, giving different aerodynamic drag and lift and in turn, a changing longitudinal motion. The high-fidelity flow simulation by RANS for construction of the aerodynamic database was carried out with varying Mach number $(M)$, angle of attack $(\alpha(t))$, and $\delta e$ for the flight path calculation based on these ranges:

$$
\begin{aligned}
M & =0.1,0.3, \text { and } 0.5 \\
\text { AoA } & =-5.0-21.0^{\circ}\left(\text { every } 2.0^{\circ}\right) \\
\text { deltae } & =-20.0-10.0^{\circ}\left(\text { every } 5.0^{\circ}\right) .
\end{aligned}
$$

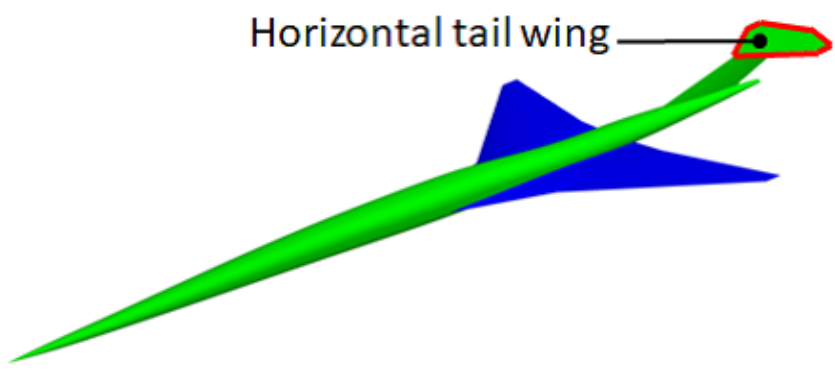

Figure 2: Illustration of SST CAD model and the horizontal tail wing as a control surface.

\section{FLIGHT PATH EVALUATION AND CON- TROL OPTIMIZATION}

The optimization procedure for the time-series flight control of SST using the efficient aerodynamics-flight dynamics is as follows:

1. Initial population with sampling $\delta e$ sequence based on the uniform random number

2. Flight simulation solving 3-DoF-EoM with aerodynamic estimation with estimation of aerodynamic coefficients

3. Calculation of $J$

4. Generation of next population by selection with constraint treatment, crossover, and mutation

The optimization procedure is iterated until $J$ is converged. Aerodynamic database to estimate aerodynamic forces in the flight simulation are constructed as follow:

1. Sampling $\delta e$ sequence by latin hypercube sampling

2. Geometries definitions

3. Computations of aerodynamic coefficients according to Eq. (4) using RANS

4. Constructions of aerodynamic database for estimations by Kriging method

\subsection{Flight Path Evaluation}




\subsubsection{Equations of Motion}

The aircraft pass was evaluated by the 3-DoF-EoM expressed in Eq. (5)through the second-order derivatives:

$$
\begin{aligned}
\frac{\mathrm{d}^{2} x}{\mathrm{~d} t^{2}} & =\frac{X}{m}-g \cos \theta \\
\frac{\mathrm{d}^{2} z}{\mathrm{~d} t^{2}} & =\frac{Z}{m}-g \sin \theta \\
\frac{\mathrm{d}^{2} \alpha}{\mathrm{d} t^{2}} & =\frac{M}{I_{y y}}-\frac{\mathrm{d} \theta}{\mathrm{d} t}
\end{aligned}
$$

where $x$ and $z$ are the respective coordinates of the aerodynamic forces $X$ and $Z$ along the body axes shown in Fig. 3; $I_{y} y$ is the moment of inertia around the axes; $m$ is mass of the aircraft and; $g$ is acceleration due to gravity.

\subsubsection{Aerodynamic Forces}

$X, Z$, and $M$ should be estimated by the time variation while EoM is solved. Derivatives of these aerodynamic forces can be predicted on the basis of geometry data and flight conditions:

$$
\begin{aligned}
X & =X 0+Q \times C_{\mathrm{X} \delta e} \delta e \\
Z & =Z 0+Q \times C_{\mathrm{Z} \delta e} \delta e \\
M & =M 0+Q \times C_{\mathrm{M} \delta e} \delta e .
\end{aligned}
$$

Where, $Q$ is the dynamic pressure (moving air's pressure). In this study, $C_{\mathrm{X} \delta e}, C_{\mathrm{X} \delta e}$, and $C_{\mathrm{X} \delta e}$ which are sensitibities of the control $\delta e$ were estimated through a semi - empirical aerodynamic prediction, USAF stability, and control DATCOM [DATCOM 1978] whereas $X 0, Z 0$, and $M 0$ which are $X 0, Z 0$, and $M 0$ at $\theta=0^{\circ}$ were evaluated using RANS. JAXA-developed Fast Aerodynamic Routine (FaSTAR) [Hashimoto et al. ] was employed for the computational fluid dynamics (CFD).

\subsubsection{Aerodynamic Estimation by Kriging Method}

The Kriging model [Donald et al. 1998, Jeong et al. 2005, Kanazaki et al. 2007] expresses the value $y\left(x_{i}\right)$ at an unknown design point $x_{i}$ based on

$$
y\left(x_{i}\right)=\mu+\epsilon\left(x_{i}\right) \quad(i=1,2, \ldots, m),
$$

where $m$ denotes the number of design variables, $\mu$ is a constant of the global model, and $\epsilon\left(x_{i}\right)$ represents a local deviation from the global model. Correlation between $\epsilon\left(x_{i}\right)$ and $\epsilon\left(x_{j}\right)$ is strongly related to the distance between the corresponding points, $x_{i}$ and $x_{j}$. Furthermore, in the model, the local deviation at an unknown point $x$ is expressed using stochastic processes. Specifically, a number of design points are calculated as sample points and then interpolated using a Gaussian random function as the correlation function to estimate the trend of the stochastic process.

\subsection{Genetic Algorithm: GA}

GAs (Fig. 4(a)) are based on the evolution of living organisms with regard to adaptation to the environment and the passing on of genetic information to the next generation[Holland 1975]. GAs can find a global optimum because they do not use function gradients, which often lead to an exact local optimum. Thus, GA is a robust and effective method that can handle highly nonlinear optimization problems involving nondifferentiable objective functions. Owing to this advantage, GAs were applied to this experimental system. The GA used in this study [Kanazaki et al. 2004, Eshelman and Schaffer 1993, Deb et al. 2002] utilizes a real-coded representation, the simulated binary crossover (SBX)[Deb et al. 2002]], and the polynomial mutation. Constraints were treated by the penalty method.

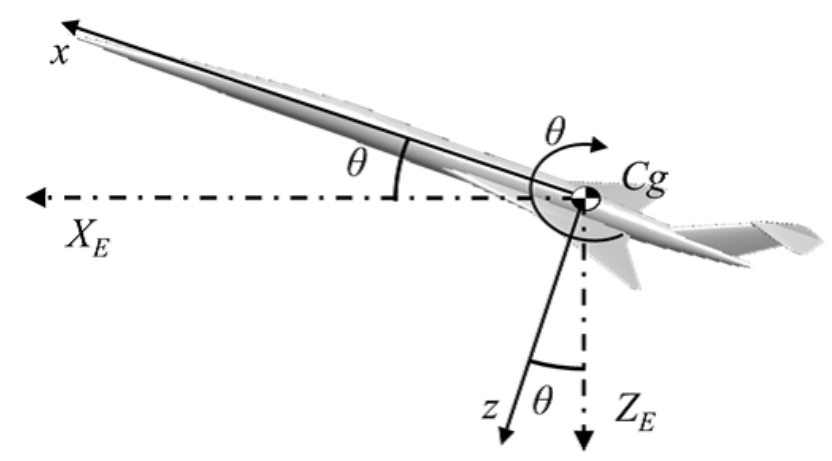

Figure 3: Coordinate system for the trajectory evaluation.

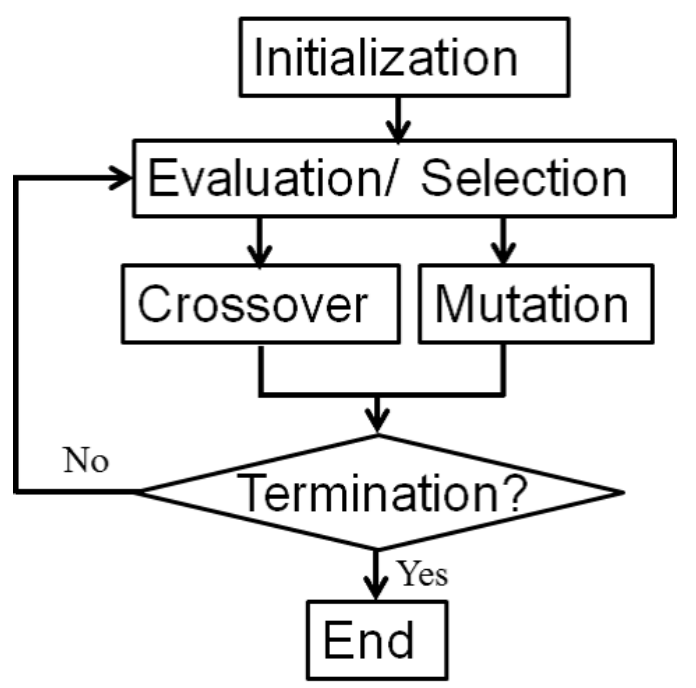

Figure 4: Schematic of the genetic algorithm (GA).

\section{RESULTS}

Herein, flight was assumed to initiate at preliminary conditions, $x(0)=0[\mathrm{~m}], z(0)=500[\mathrm{~m}], u(0)=140.04[\mathrm{~m} / \mathrm{s}]$, $\theta(0)=0.0\left[^{\circ}\right]$, initial angular velocity $q(0)=0.0\left[{ }^{\circ}\right]$, and $\theta(0)=-3.4\left[{ }^{\circ}\right]$. The population size was 10 and the GA was executed for 40 generations. $J$ was evaluated using the efficient aerodynamic-flight dynamics during GA execution. Figure 5 presents the flowfields obtained by varying the angles of attack and Mach number, i.e., $M=0.3$.

\subsection{Design Exploration Results}

Figure 6(a) shows the history of $J$ from the feasible solutions by GA. The minimum $J$ by feasible solutions in each 

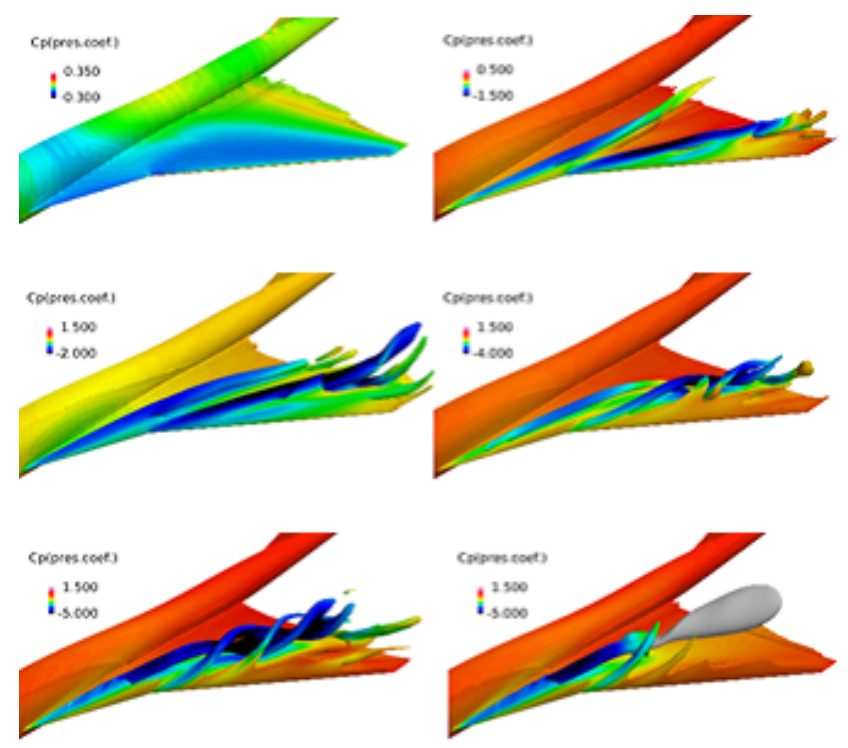

Figure 5: Floweld variation with changing angles of attack around the SST model.

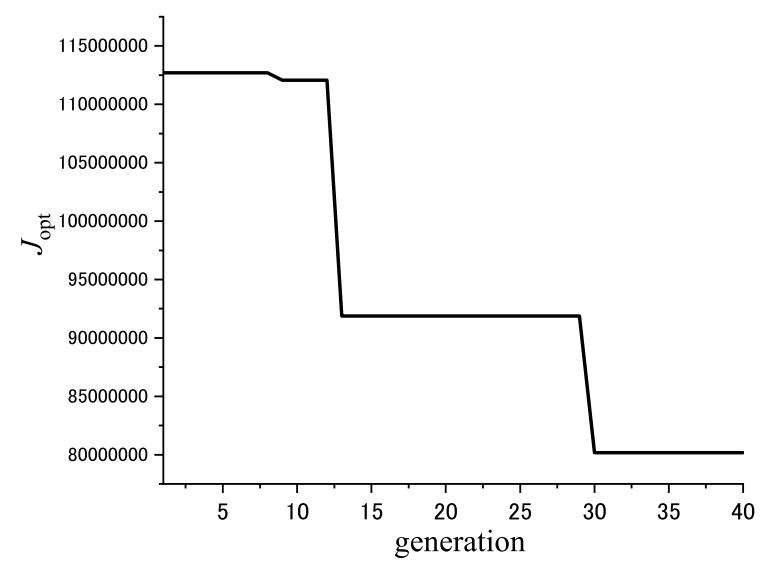

(a)

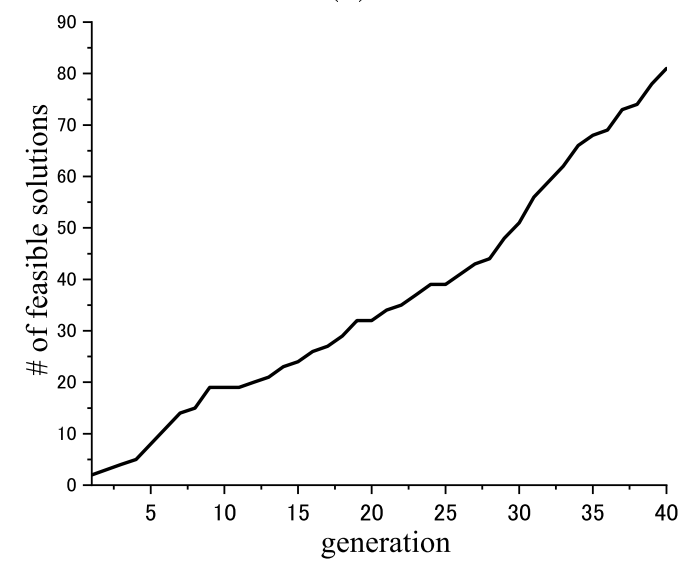

(b)

Figure 6: Results of GA.(a)History of $J$ and (b)History of the number of feasible solutions

generation was gradually minimized, along with a corresponding gradual increase in the number of feasible solutions as shown in Fig. 6(b). Thus, optimization by GA was successfully implemented for the time-series flight control problem.

\subsection{Feature of Optimum Solutions}

Figure 7 describes the flight paths of solutions with the smallest and the largest $J$ among the feasible solutions. The flight having the minimum $J$ reached approximately 2500.0 $\mathrm{m}$, whereas the flight with the maximum $J$ reached approximately $4000.0 \mathrm{~m}$. Moreover, the flight with the minimum $J$ showed a decently linear curve (Fig. 7(a)), thus, giving better flight efficiency than that with maximum $J$.

Figure 8 shows the best five time history curves of the $\theta(t)$ and $w(t)$ obtained by the optimization. In contrast, Fig. 9 shows the worst five time history curves. In comparison, both flights showed that $\theta(t)$ was approximately $15^{\circ}$ at the termination $t_{\mathrm{t}}$ to satisfy the constraint by the reduction of $w(t)$, as shown in Figs. 8(b) and 9(b). Nonetheless, each solution revealed a non-monotonic variation for $\theta(t)$, whose minimum falls at $t=5$ - 10 [s.]

The remarkable difference between the best and the worst solutions of $J$ was the time history curve of $x(t)$ and $u(t)$. For the best solution, the aircraft should land shortly at $w(t)<5[\mathrm{~m} / \mathrm{s}]$, in which the time variation of $w(t)$ speed rapidly decelerates (Fig. 8). Otherwise, the worst solution is for the aircraft to satisfy the constraint $w(t)<5[\mathrm{~m} / \mathrm{s}]$, before continually flying horizontally at higher $u(t)$. In this case, $J$ gets large as flight time and the flight distance increase.

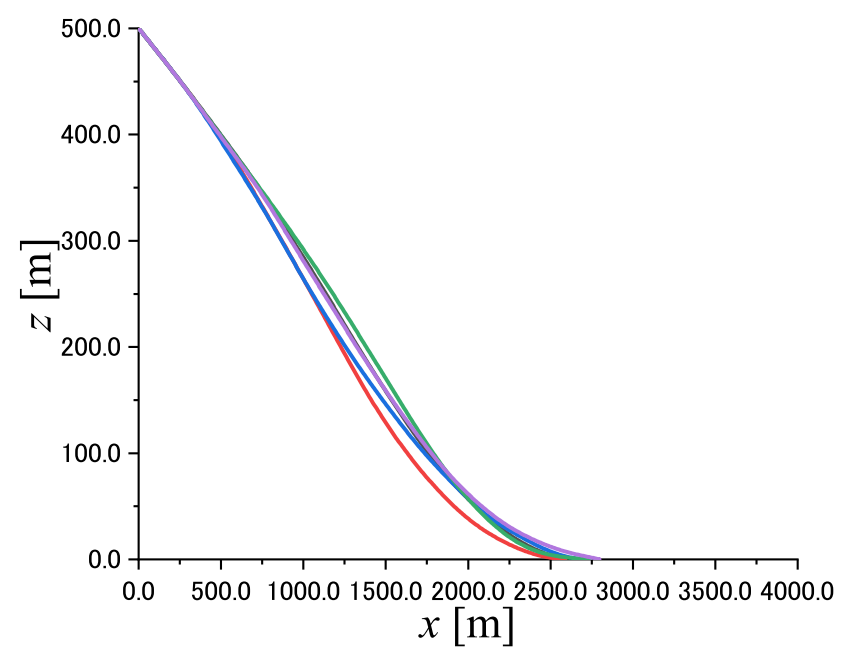

(a)

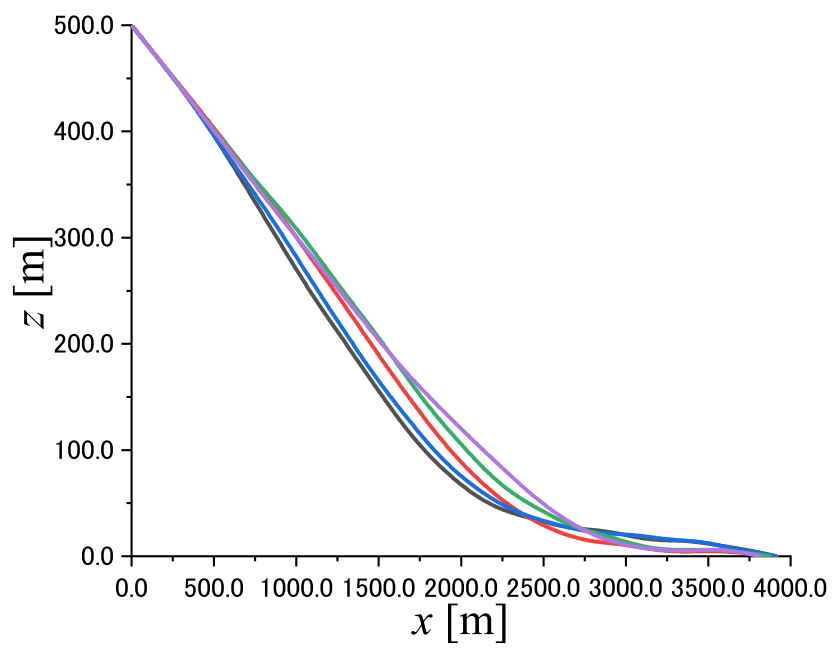

(b)

Figure 7: Comparison of flight paths obtained by GA. (a) Flight path with the best five $J$ and (b)fight path with the worst five $J$. 


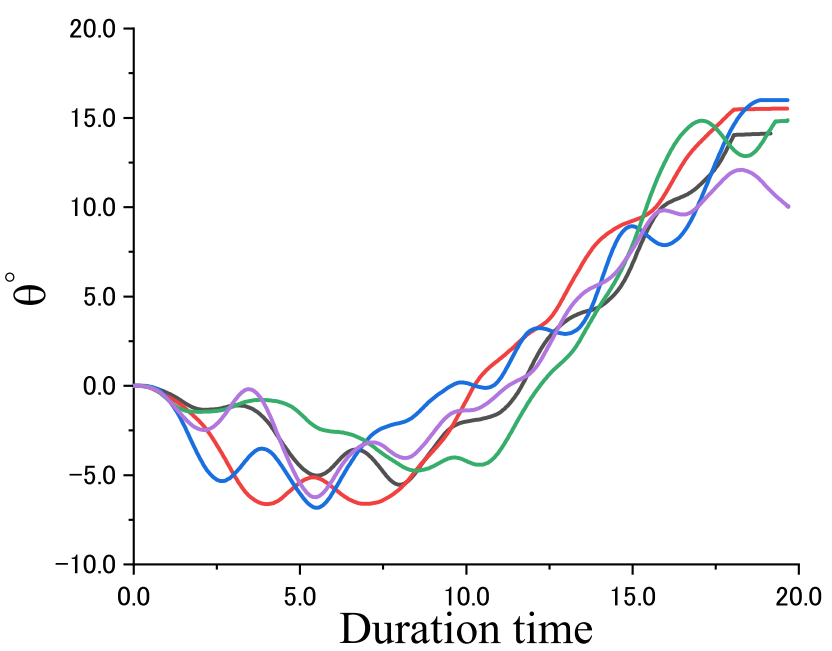

(a)

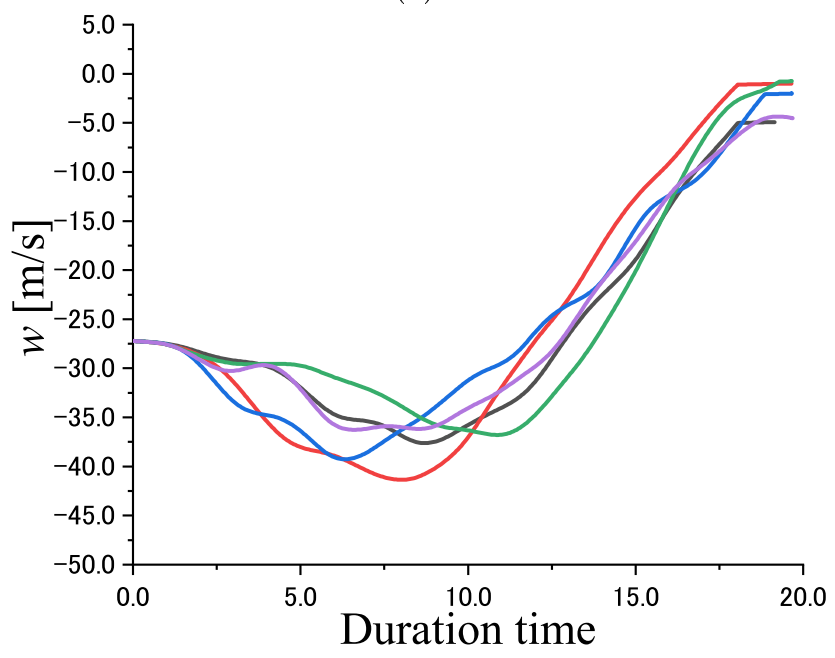

(b)

Figure 8: Comparison of time history curves of the best five flight individual properties of (a) $\theta(t)$ and (b) $w(t)$.

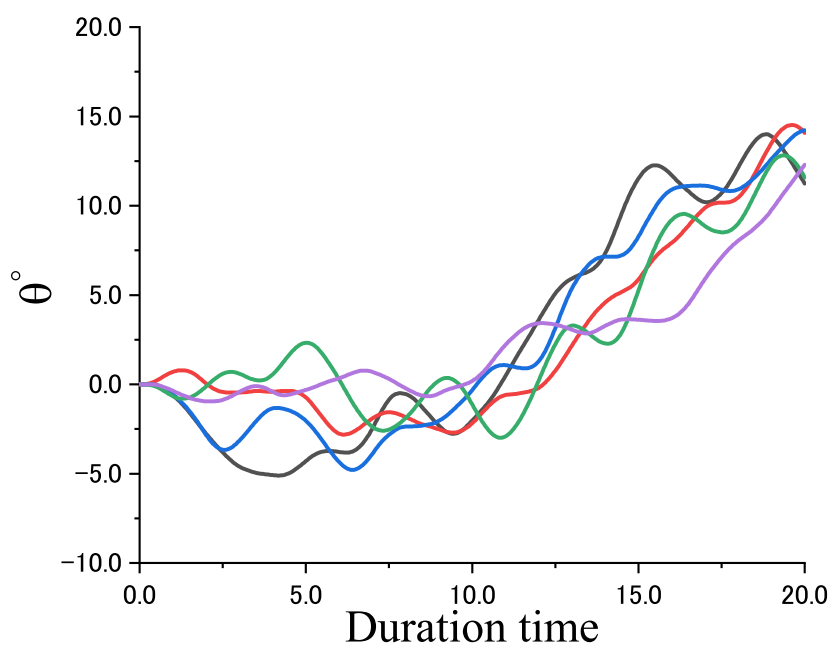

(a)

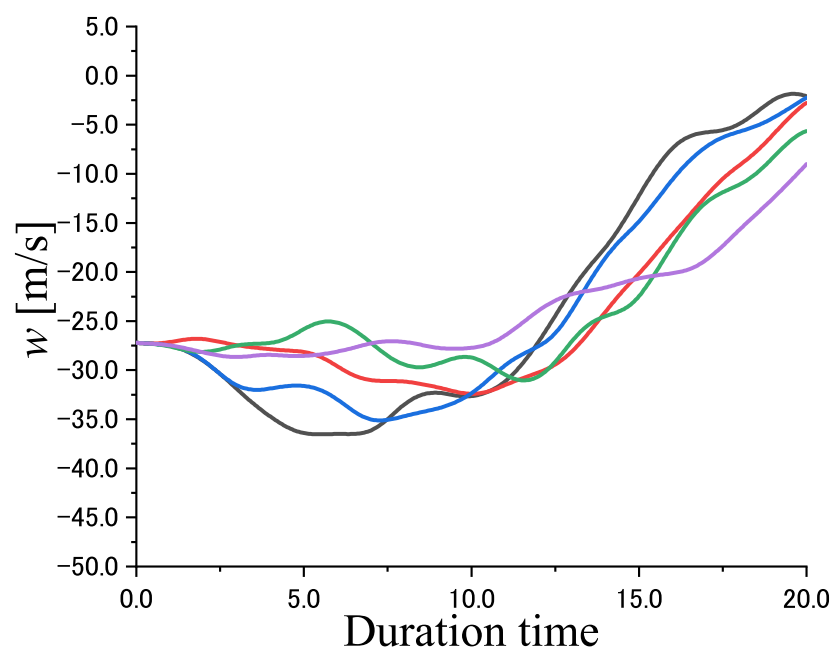

(b)

Figure 9: Comparison of time history curves of the worst five flight properties of (a) $\theta(t)$ and (b) $w(t)$.

\section{CONCLUSIONS}

We conducted a time-series flight path and control optimization for the landing descent of a delta-winged supersonic transport (SST) having horizontal wings range, using genetic algorithm (GA). A flight simulation was performed on the basis of solving a 3-degree-of-freedom EoM while the aerodynamic forces were predicted from an aerodynamic database through Kriging method. A high-fidelity flow solver was used to determine aerodynamic calculations for the complex flowfields around the SST. Moreover, the aerodynamic control force was obtained as the deflection angle of the horizontal tail wing varied. Aerodynamic estimation by Kriging was effective in the efficient aerodynamic - flight dynamics simulation, and could be apply for practical computational cost via the GA.

The design problem assumed an SST landing at $500.0 \mathrm{~m}$ altitude given an initial speed, with the vertical speed after landing (altitude $=0 \mathrm{~m}$ ) treated as a constraint. Through GA, we found an optimal flight path after the aircraft satisfied the constraint. From the design results, we obtained the correlation between pitch angle and the vertical speed. In particular, pitch angle increases to reduce the vertical speed with increasing aerodynamic drag. A high-fidelity flow simulation required to accurately evaluate such aerodynamics described by a high pitch angle and angle of attack, could be effectively represented by the proposed aerodynamics-flight dynamics simulation for global optimization of the flight path and control of arbitrary aircraft.

\section{ACKNOWLEDGMENT}

The authors are grateful to Dr. Y. Makino for sharing the geometry data of JAXA's SST model. This work was supported by JSPS KAKENHI Grant Number 16K06888.

\section{REFERENCES}

[1] [DATCOM 1978] 1978. R. D. Fink. 2002. USAF Stability and Control DATCOM, Flight Control Division. AFWALTR-83-3048, (1978). 
[2] [Deb et al. 2002] K. Deb, A. Pratap, S. Agarwal, and T. Meyarivan. 2002. A Fast and Elitist Multiobjective Genetic Algorithm: NSGA-II. IEEE Transactions on Evolutionary Computation 6, 2 (2002), 182_197. https://doi.org/10.1109/4235.996017

[3] [Donald et al. 1998] R. J. Donald, S. Matthias, and J. W. William. 1998. Efficient Global Optimization of Expensive Black-Box Function. Journal of Global Optimization 13 (1998), 455_492.

[4] [Ericsson 1996] L. E. Ericsson. 1996. Pitch rate effects on delta wing vortex breakdown. J. Aircraft 33, 3 (May 1996), 639_642. https://doi.org/10.2514/2.2165

[5] [Eshelman and Schaffer 1993] L.J. Eshelman and J.D. Schaffer. 1993. Real-Coded Genetic Algorithms and Interval-Schemata. Foundations of Genetic Algorithms2, 187-202. https://doi.org/10.1016/B978-0-08-094832$4.50018-0$

[6] [Hashimoto et al.] A. Hashimoto, K. Murakami, T. Aoyama, K. Ishiko, M. Hishida, M. Sakashita, and P. Lahur. 2012. Toward the Fastest Unstructured CFD Code 'FaSTAR'. AIAA 2012-1075. https://doi.org/10.2514/6.2012-1075

[7] [Holland 1975] J. H. Holland. 1975. Adaptation in Natural and Artificial Systems. University of Michigan Press Ann Arbor (1975).
[8] [Jeong et al. 2005] S. Jeong, M. Murayama, and K. Yamamoto. 2005. Efficient Optimization Design Method Using Kriging Model. Journal of Aircraft 42, 2 (2005), 413420. https://doi.org/10.2514/1.6386

[9] [Kanazaki et al. 2004] M. Kanazaki, S. Obayashi, and K. Nakahashi. 2004. Exhaust Manifold Design with Tapered Pipes Using Divided Range MOGA. Engineering Optimization, Taylor \& Francis 36, 2 (2004), 149_164. https://doi.org/10.1080/03052150310001639281

[10] [Kanazaki and Othmam 2016] M. Kanazaki and N Othmam. 2016. Time-series Optimization Methodology and Knowledge Discovery of Descend Trajectory for Civil Aircraft. https://doi.org/10.1109/CEC.2016.7743885

[11] [Kanazaki et al. 2007] M. Kanazaki, K. Tanaka, S. Jeong, and K. Yamamoto. 2007. Multi-Objective Aerodynamic Exploration of Elements' Setting for High-Lift Airfoil Using Kriging Model. J Aircraft 44, 3 (2007), 858_864. https://doi.org/10.2514/1.25422 https://doi.org/10.1109/CEC.2016.7743885

[12] [Liu et al. 2018] J. Liu, H. Sun, and Z. Xi. 2018. Vortex Breakdown Flows Around a Double-Delta Wing During Pitching Motion Based on DDES. 313_324.

[13] [Srigrarom and Lewpiriyawong 2007] S. Srigrarom and N. Lewpiriyawong. 2007. Controlled Vortex Breakdown on Modified Delta Wings. J. Visualizationt 10, 3 (Aug. 2007), 299_307. https://doi.org/10.1007/BF03181697 